\title{
Pentapartitioned Neutrosophic Generalized Semi-closed Sets
}

Radha $\mathrm{R}^{1}$ and Stanis Arul Mary A*,2

Received: 02 April 2021/ Accepted: 18 May 2021/ Published online: 18 June 2021

(C)Sacred Heart Research Publications 2017

\begin{abstract}
The aim of the paper is to introduce and investigate the concepts of Pentapartitioned neutrosophic generalized semi-closed sets (PNGSCS) and Pentapartitioned neutrosophic generalized semi-open sets (PNGSOS) in Pentapartitioned neutrosophic topological space.

Key words: Pentapartitioned Neutrosophic Set, Pentapartitioned Neutrosophic Generalized Semi-Closed Sets, Pentapartitioned Neutrosophic Generalized Semi-Open Sets.
\end{abstract}

AMS classification: 03E72, 03B52

\section{Introduction}

The fuzzy set was initiated by Zadeh [18] in 1965, where each element had a degree of membership. The neutrosophic set was introduced by Smarandache [16] and explained, neutrosophic set is a generalization of intuitionistic fuzzy set. They introduced neutrosophic topological space as a generalization of intuitionistic fuzzy topological space and a neutrosophic set besides the degree of membership, the degree of indeterminacy and therefore the degree of non-membership of each element. For the past few years, many researchers were going on in neutrosophic topological spaces and many concepts in intuitionistic fuzzy topology were extended to neutrosophic topology. Rama Malik and Surpati Pramanik [11 introduced Pentapartitioned neutrosophic set and its properties. Here indeterminacy is split into three parts as contradiction, ignorance and unknown membership function. Also we introduced the concept of Penta partitioned neutrosophic Pythagorean set [6] and establish a number of its properties in our previous work.

The concepts of pentapartitioned neutrosophic semi-open sets, pentaparti-

\footnotetext{
1,2 Department of Mathematics, Nirmala College for Women, Coimbatore, India.

Email: ${ }^{1}$ radharmat2020@gmail.com, ${ }^{*}, 2$ kathirsubbu1987@gmail.com
} 
ISSN: 2456-8686, 5(1), 2021:123-131

https://doi.org/10.26524/cm99

tioned neutrosophic semi-closed sets, pentapartitioned neutrosophic semi-interior and pentapartitioned neutrosophic semi-closure in pentapartitioned neutrosophic topological spaces were introduced by R. Radha and A. Stanis Arul Mary[10]. In this paper, we introduce the definitions of pentapartitioned neutrosophic generalized semi-closed sets and pentapartitioned neutrosophic generalized semi-open sets in pentapartitioned neutrosophic topological spaces and We study some of their basic properties with examples.

\section{Preliminaries}

Definition 2.1 [1] Let X be a universe. A Pentapartitioned neutrosophic set A on $\mathrm{X}$ is an object of the form

$$
A=\left\{<x, T_{A}, C_{A}, I_{A}, U_{A}, F_{A}>: x \in X\right\}
$$

Where $T_{A}+F_{A}+I_{A}+C_{A}+U_{A} \leq 5$.

Here, $T_{A}(x)$ is the truth membership, $C_{A}(x)$ is contradiction membership, $U_{A}(x)$ is ignorance membership, $F_{A}(x)$ is the false membership and $I_{A}(x)$ is an unknown membership.

Definition 2.2 [6] A $\mathrm{PN}$ set $\mathrm{A}$ is contained in another $\mathrm{PN}$ set $\mathrm{B}$ (i.e) $\mathrm{A} \subseteq B$ iff $T_{A} \leq T_{B}, C_{A} \leq C_{B}, I_{A} \geq I_{B}, U_{A} \geq U_{B}$ and $F_{A} \geq F_{B}$

Definition 2.3 6] The complement of a PN set $(\mathrm{H}, \mathrm{A})$ on $\mathrm{R}$ is defined as $H^{c}(x)=\left\{<x, F_{A}, U_{A} 1-I_{A}, C_{A}, T_{A}>: x \in R\right\}$ and is denoted by $(H, A)^{c}$.

Definition 2.4 [6] Let $\mathrm{R}$ be a non-empty set, $A=\left\langle x, T_{A}, C_{A}, I_{A}, U_{A}, F_{A}\right\rangle$ and $B=\left\langle x, T_{B}, C_{B}, I_{B}, U_{B}, F_{B}\right\rangle$ are two PN sets. Then

$A \cup B=\left\langle x, \max \left(T_{A}, T_{B}\right), \max \left(C_{A}, c_{B}\right), \min \left(I_{A}, I_{B}\right), \min \left(U_{A}, U_{B}\right), \min \left(F_{A}, F_{B}\right)\right\rangle$

$A \cap B=\left\langle x, \min \left(T_{A}, T_{B}\right), \min \left(C_{A}, c_{B}\right), \max \left(I_{A}, I_{B}\right), \max \left(U_{A}, U_{B}\right), \max \left(F_{A}, F_{B}\right)\right\rangle$

Definition 2.5 [6] A PN set (F, A) over the universe X is said to be empty PN set $0_{X}$ with respect to the parameter $\mathrm{A}$ if

$T_{F(e)}=0, C_{F(e)}=0, I_{F(e)}=1, U_{F(e)}=1, F_{F(e)}=1, \forall x \in X, \forall e \in A$. It is denoted by $0_{X}$. 
Definition 2.6 [6] A PN set (F, A) over the universe $\mathrm{R}$ is said to be universe Pentapartitioned neutrosophic set with respect to the parameter A if $T_{F(e)}=1, C_{F(e)}=1, I_{F(e)}=0, U_{F(e)}=0, F_{F(e)}=0, \forall x \in X, \forall e \in A$. It is denoted by $1_{R}$.

Definition 2.7 [10] Let A be PN set of a pentapartitioned neutrosophic topological space [PNTS] $\mathrm{X}$. Then $\mathrm{A}$ is said to be PN semi-open set [PNSO set] of $\mathrm{X}$ if there exists a set PNO such that $P N O(A) \subseteq A \subseteq P N C l(P N O(A))$.

Definition 2.8 [10] A subset A in a PNTS X is PNSO set if and only if $A \subseteq P N C l(P N \operatorname{Int}(A))$.

Definition 2.9 [10] A PNPS A is called Pentapartitioned neutrosophic semi-closed set $[\mathrm{PNSC}$ set] if the complement of $\mathrm{C}(\mathrm{A})$ is a PNSO set.

\section{Pentapartitioned Neutrosophic Generalized Semi-closed Sets}

Definition 3.1 A Pentapartitioned neutrosophic set [PNS] A of a Pentapartitioned neutrosophic topological space $\operatorname{PNTS}(R, \tau)$ is called a Pentapartitioned neutrosophic generalized semi-closed set [PNGSC set] if $\operatorname{PNSCl}(A) \subseteq U$, whenever $A \subseteq U$ and $\mathrm{U}$ is a Pentapartititioned neutrosophic open (PNO) set.

Example 3.2 Let $R=\{a\}$ with $\tau=\left\{0_{R}, A, B, C, D, E, F, 1_{R}\right\}$

where $\mathrm{A}=\{0.1,0.6,0.5,0.2,0.6\}, \mathrm{B}=\{0.4,0.6,0.1,0.2,0.4\}, \mathrm{C}=\{0.4,0.1,0.5 .0 .6,0.4\}$,

$\mathrm{D}=\{0.1,0.4,0.7,0.5,0.6\}, \mathrm{E}=\{0.4,0.6,0.5,0.2,0.4\}$ and $\mathrm{F}=\{0.4,0.4,0.5,0.5,0.4\}$.

Then the PNGSO sets are $0_{R}, \mathrm{~A}, \mathrm{~B}, \mathrm{C}, \mathrm{D}, \mathrm{E}, \mathrm{F}, \mathrm{C}(\mathrm{A}), \mathrm{C}(\mathrm{D}), \mathrm{C}(\mathrm{F}), 1_{R}$.

Let us take $\mathrm{M}=\{0.4,0.2,0.5,0.1,0.3\}$. Then $\mathrm{M}$ is PNGSC set.

Definition 3.3 A PNPS A in R is called PN generalized semi-open set [PNGSO set] in $\mathrm{R}$ if $\mathrm{C}(\mathrm{A})$ is PNGSC set in $\mathrm{R}$. That is, $U \subseteq P N S \operatorname{Int}(A)$, whenever $A \subseteq U$ and $\mathrm{U}$ is a $\mathrm{PNC}$ set.

Definition 3.4 Let $(R, \tau)$ be a PNTS. Then a PN subset $\mathrm{P}$ of the PNTS $\mathrm{R}$ is said to be $\mathrm{PN}$ regular open if $\mathrm{P}=\mathrm{PNInt}(\mathrm{PNCl}(\mathrm{P}))$ and $\mathrm{PN}$ regular closed if $\mathrm{A}$ $=\operatorname{PNCl}(\mathrm{PNInt}(\mathrm{P}))$ 
Theorem 3.5 Every PN closed set in PN topological space $(\mathrm{R}, \tau)$ is a PN generalized semi-closed set.

Proof : Let A be a PNC set in PN topological space $(R, \tau)$. Let $A \subseteq U$ and $\mathrm{U}$ be a $\mathrm{PN}$ open set in $\mathrm{R}$. Then by Definition, $\mathrm{A}=\operatorname{PNCl}(\mathrm{A})$. We know that $\mathrm{PNSCl}(\mathrm{A}) \subseteq \mathrm{PNCl}(\mathrm{A})$, then we get $\mathrm{PNSCl}(\mathrm{A}) \subseteq P N C l(A)=A \subseteq U$. Hence $\mathrm{A}$ is a $\mathrm{PN}$ generalized semi-closed set in $\mathrm{R}$.

Example 3.6 From the above (3.2), M is PNGSC set. but not PNC set.

Theorem 3.7 Every PNSC set in the PNTS $(R, \tau)$ is a PNGSC set.

Proof: Let P be a PNSC set in the PNTS R. Let $P \subseteq U$ and $U$ be a PN open set in $\mathrm{R}$. Since $\mathrm{P}$ is $\mathrm{PN}$ semi-closed set, $\mathrm{PNInt}(\mathrm{PNCl}(\mathrm{P})) \subseteq \mathrm{P}$. Therefore $\mathrm{PNSCl}(\mathrm{P}) \subseteq U, P \subseteq \mathrm{U}$ and $\mathrm{U}$ is a $\mathrm{PN}$ open set. Hence $\mathrm{P}$ is a PNGSC set in $\mathrm{R}$.

Example 3.8 From the above Example (3.2), M is PNGSC set but not PNSC set.

Theorem 3.9 If $\mathrm{P}$ and $\mathrm{Q}$ are PNGSC sets, then their intersection is also a PNGSC set.

Proof: Let $\mathrm{P}$ and $\mathrm{Q}$ are PNGSC sets. If $P \cap Q \subseteq U$ and $\mathrm{U}$ is $\mathrm{PN}$ open set, then $P \subseteq U$ and $Q \subseteq U$. Since P and $\mathrm{Q}$ are PNGSC sets, $P N S C l(P) \subseteq U$ and $P N S C l(Q) \subseteq U$. Hence $P N S C l(P) \cap P N S C l(Q) \subseteq U$. By theorem, $P N C l(P \cap Q) \subseteq P N S C l(P) \cap P N S C l(Q) \subseteq U$. Thus $P \cap Q$ is PNGSC set.

Remark 3.10 Union of any two PNGSC sets in $(\mathrm{R}, \tau)$ need not be a PNGSC set, as seen from the following example.

Example 3.11 From Example $(3.2), B^{C}$ and D are PNGSC sets but their union $\mathrm{S}$ is not PNGSC set.

Theorem 3.12 If $\mathrm{P}$ is a $\mathrm{PNGSC}$ set in $\mathrm{R}$ and $P \subseteq Q \subseteq P N S C l(P)$, then $\mathrm{Q}$ is a PNGSC set in R.

Proof: Let $\mathrm{U}$ be a $\mathrm{PNO}$ set in $\mathrm{R}$ such that $Q \subseteq U$. Since $P \subseteq Q, P \subseteq U$. Again since $\mathrm{P}$ is a PNGSC set, $\operatorname{PNSCl}(P) \subseteq U$. By hypothesis, $Q \subseteq \operatorname{PNSCl}(P)$. We know that $P N S C l(Q) \subseteq P N S C l(P N S C l(P))=P N S C l(P)$. That is 
$P N S C l(Q) \subseteq P N S C l(P)$. This implies that $P N S C l(q) \subseteq U$. Hence Q is a PNGSC set in $\mathrm{X}$.

Theorem 3.13 A PN set $\mathrm{P}$ of a PN topological space $(R, \tau)$ is a PNGSC set iff $P N S C l(P) \subseteq \mathrm{Q}$ where $\mathrm{Q}$ is a $\mathrm{PNO}$ set and $P \subseteq Q$.

Proof : Assume that $\mathrm{P}$ is a PNGSC set in X. Let $\mathrm{Q}$ be a PNO set in $\mathrm{R}$ such that $P \subseteq Q$. Then $\mathrm{C}(\mathrm{Q})$ is a $\mathrm{PNC}$ set in $\mathrm{R}$ such that $C(Q) \subseteq C(P)$. Since $\mathrm{C}(\mathrm{P})$ is a PNGSO set, $C(Q) \subseteq P N S I n t(C(P))$. By Proposition 6.2 (ii) [5], PNSInt $(\mathrm{C}(\mathrm{P}))$ $=\mathrm{C}(\mathrm{PNSCl}(\mathrm{P}))$. Therefore $C(Q) \subseteq C(P N S C l(P))$ implies that $P N S C l(P) \subseteq Q$. Conversely, assume that $P N S C l(P) \subseteq Q$ where $\mathrm{Q}$ is a $\mathrm{PNO}$ set and $P \subseteq Q$. Then $C(Q) \subseteq C(P N S C l(A))$ where $\mathrm{C}(\mathrm{Q})$ is a $\mathrm{PNC}$ set and $C(Q) \subseteq P N S \operatorname{Int}(C(\mathrm{P}))$. Therefore $\mathrm{C}(\mathrm{P})$ is a PNGSO set. This implies that $\mathrm{P}$ is a PNGSC set.

Theorem 3.14 A PN set $\mathrm{P}$ of a PNTS $(R, \tau)$ is a PNGSC set iff $P N \operatorname{Int}(P N C l(P) \subseteq Q$ whenever $\mathrm{Q}$ is a $\mathrm{PNO}$ set and $P \subseteq Q$.

Proof : Assume that $\mathrm{P}$ is a PNGSC set in R. Let $\mathrm{Q}$ be a $\mathrm{PNO}$ set in $\mathrm{R}$ and $P \subseteq Q$. Then $\mathrm{C}(\mathrm{Q})$ is a PNC set in $\mathrm{R}$ such that $C(Q) \subseteq C(P)$. Since $\mathrm{C}(\mathrm{P})$ is a PNGSO set, $C(Q) \subseteq P N S \operatorname{Int}(C(P))$. Therefore $C(Q) \subseteq P N C l(P N \operatorname{Int}(C(P)))$.

Hence $C(Q) \subseteq C(P N \operatorname{Int}(P N C l(P)))$. This implies that PNInt $(P N C l(P)) \subseteq Q$. Conversely, let $\mathrm{P}$ be $\mathrm{PN}$ set of $\mathrm{R}$ and $\operatorname{PNInt}(\operatorname{PNCl}(P) \subseteq Q$ whenever $\mathrm{Q}$ is a $\mathrm{PNO}$ set and $P \subseteq Q$. Then $C(Q) \subseteq C(P)$ and $\mathrm{C}(\mathrm{Q})$ is PNC set. By hypothesis $C(Q) \subseteq$ $C(P N \operatorname{Int}(P N C l(P)))$.

Hence $C(Q) \subseteq P N C l(P N \operatorname{Int}(C(P)))$ implies that $C(Q) \subseteq P N \operatorname{SInt}(C(P)))$. So that $\mathrm{C}(\mathrm{P})$ is PNGSO set. Hence $\mathrm{P}$ is PNGSC set of R.

\section{Pentapartitioned Neutrosophic Generalized Semi-open Sets}

In this section, we study the concepts of Pentapartitioned neutrosophic generalized semi-open sets(PNGSO) and some of their basic properties.

Definition 4.1 The family of all PNGSC set (resp,PNGSO set) of a PN topological space $(R, \tau)$ will be denoted by PNGSC (R) ( resp. PNGSO (R)).

Example 4.2 From Example (3.2), Let $\mathrm{H}=\{0.3,0.1,0.5,0.2,0.4\}$.Hence $\mathrm{H}$ is $\mathrm{PN}$ generalized semi-open set. 
Theorem 4.3 Every PNO set in PNTS $(R, \tau)$ is a PNGSO set.

Proof: Let $\mathrm{P}$ be a PNO set in PNTS R. Then by Definition, $\mathrm{P}=\operatorname{PNInt}(\mathrm{P})$. We know that $P N \operatorname{Int}(P) \subseteq P N S \operatorname{Int}(P) \subseteq P$. Therefore $\mathrm{P}=\operatorname{PNSInt}(\mathrm{P})$. Hence $\mathrm{P}$ is a PNGSO set in R.

Example 4.4 From the above Example (3.2), H is PNGSO set but not PNO set.

Theorem 4.5 Every PNSO set in $\operatorname{PNPTS}(R, \tau)$ is a PNGSO set.

Proof : Let $\mathrm{P}$ be a PNSO set in PNTS R. Let $U \subseteq P$ and $\mathrm{U}$ be a PNO set in R. Since $\mathrm{P}$ is $\mathrm{PNSO}$ set, $P \subseteq P N C l(P N \operatorname{Int}(P)$. This implies that $P \subseteq P N \operatorname{Sint}(P)=P \cap P N C l(P N \operatorname{Int}(P))$. Since $U \subseteq P, U \subseteq P N \operatorname{Sint}(P)$. Therefore $U \subseteq P N S \operatorname{Int}(P), U \subseteq P$ and $\mathrm{U}$ is a $\mathrm{PNC}$ set. Hence $\mathrm{P}$ is a PNGSO set in $\mathrm{R}$.

Example 4.6 From the Example (3.2), H is PNGSO set but not PNSO set.

Theorem 4.7 If $\mathrm{P}$ and $\mathrm{Q}$ are PNGSO sets, then $P \cup Q$ is also a PNGSO set.

Proof : Let $\mathrm{P}$ and $\mathrm{Q}$ are PNGSO sets.If $U \subseteq P \subseteq Q$ and $\mathrm{U}$ is a PNC set, then $U \subseteq P$ and $U \subseteq Q$. Since $\mathrm{P}$ and $\mathrm{Q}$ are PNGSO sets, $U \subseteq P N S \operatorname{Pnt}(P)$ and $U \subseteq P N S \operatorname{Int}(Q)$. Hence $U \subseteq P N S \operatorname{Int}(P) \cup P N \operatorname{SInt}(Q)$. We know that $P N S \operatorname{Int}(P \cup Q) \supseteq P N S \operatorname{Int}(P) \cup P N S \operatorname{Int}(Q) \supseteq U$. This implies that $U \subseteq P N S \operatorname{Int}(P \cup Q)$. Therefore $U \subseteq P N \operatorname{SInt}(P \cup Q), U \subseteq P \cup Q$ and $\mathrm{U}$ is PNC set. Thus $P \cup Q$ is PNGSO set.

Remark 4.8 Intersection of any two PNGSO sets in $(R, \tau)$ need not be a PNGSO set, as seen from the following example.

Example 4.9 From Example (3.2), we consider the two PNGSO sets C (A) and C $(\mathrm{F})$. Their union is PNGSO set but their intersection is not PNGSO set.

Theorem 4.10 If $\mathrm{P}$ is a PNGSO in $\mathrm{R}$ and if $P N S \operatorname{Int}(P) \subseteq Q \subseteq P$, then $\mathrm{Q}$ is a PNGSO set in R.

Proof : Let $\mathrm{P}$ be a PNGSO set in R. Since PNSInt $(P) \subseteq Q \subseteq P$ and we have $C(P) \subseteq C(Q) \subseteq C(P N S \operatorname{Int}(P))=P N S C l(C(P)))$. Again since $\mathrm{C}(\mathrm{P})$ is a PNGSC 
set and by theorem we get $\mathrm{C}(\mathrm{Q})$ is a PNGSC set in R. Hence Q is a PNGSO set in $\mathrm{R}$.

Theorem 4.11 A PN set P of a PN topological space $(R, \tau)$ is a PNGSO set if and only if $Q \subseteq P N \operatorname{Sint}(P)$ where $\mathrm{Q}$ is a $\mathrm{PN}$ closed set and $Q \subseteq P$.

Proof : Assume that P is a PNGSO set in R. Let $\mathrm{Q}$ be a PN closed set in $\mathrm{R}$ such that $Q \subseteq P$. Then $\mathrm{C}(\mathrm{Q})$ is a $\mathrm{PNO}$ set in $\mathrm{R}$ such that $C(P) \subseteq C(Q)$. Since $\mathrm{C}(\mathrm{P})$ is a PNGSC set, $P N S C l(C(P)) \subseteq C(Q)$.

Then $\mathrm{PNSCl}(\mathrm{C}(\mathrm{P}))=\mathrm{C}(\mathrm{PNSInt}(\mathrm{P}))$. Therefore $C(P N \operatorname{SInt}(P)) \subseteq C(Q)$ implies that $Q \subseteq P N S \operatorname{Int}(P)$. Conversely, assume that $Q \subseteq P N \operatorname{SInt}(P)$ where $\mathrm{Q}$ is a $\mathrm{PNC}$ set and $Q \subseteq P$. Then $C(P N S \operatorname{Int}(P)) \subseteq C(Q)$ where $\mathrm{C}(\mathrm{Q})$ is a $\mathrm{PNO}$ set and $P N S C l(C(P)) \subseteq C(Q)$. Therefore $\mathrm{C}(\mathrm{P})$ is a PNGSC set. This implies that $\mathrm{P}$ is a $\mathrm{PNGSO}$ set.

Theorem 4.12 A PN set $\mathrm{P}$ of a $\operatorname{PNTS}(R, \tau)$ is a PNGSO set if and only if $Q \subseteq P N C l(P N \operatorname{Int}(P))$ whenever $\mathrm{Q}$ is a $\mathrm{PNC}$ set and $Q \subseteq P$.

Proof : Assume that $\mathrm{P}$ is a PNGSO set in R. Let $\mathrm{Q}$ be a $\mathrm{PNC}$ set in $\mathrm{R}$ and $Q \subseteq P$. Then $\mathrm{C}(\mathrm{Q})$ is a $\mathrm{PNO}$ set in $\mathrm{R}$ such that $C(P) \subseteq C(Q)$.

Since $\mathrm{C}(\mathrm{P})$ is a PNGSC set, $P N S C l(C(P)) \subseteq C(Q)$. Therefore $P N \operatorname{Int}(P N C l(C(P))) \subseteq C(Q)$. Hence $C(P N C l(P N \operatorname{Int}(P)) \subseteq C(Q)$. This implies that $Q \subseteq P N C l(P N \operatorname{Int}(P))$. Conversely, let $\mathrm{P}$ be $\mathrm{PN}$ set of $\mathrm{R}$ and $Q \subseteq P N C l(P N \operatorname{Int}(P))$ whenever $\mathrm{Q}$ be PNC set and $Q \subseteq P$. Then $C(P) \subseteq C(Q)$ and $\mathrm{C}(\mathrm{Q})$ is $\mathrm{PNO}$ set. By hypothesis $C(\operatorname{PNCl}(\operatorname{PNInt}(P))) \subseteq C(Q)$. Hence $P N \operatorname{Int}(P N C l(C(P))) \subseteq C(Q)$ implies that $P N S C l(C(P)) \subseteq C(Q)$. So that $\mathrm{C}(\mathrm{P})$ is PNGSC set. Hence $\mathrm{P}$ is PNGSO set of R.

\section{Conclusions}

The concepts of pentapartitioned neutrosophic generalized semi-closed sets, pentapartitioned neutrosophic generalized semi-open sets, and their properties in pentapartitioned neutrosophic topological spaces were studied in this paper. In the future, we will extend these pentapartitioned neutrosophic topology concepts to include pentapartitioned neutrosophic generalized semi-continuous, pentapartitioned neutrosophic semi-generalized continuous, and pentapartitioned neutrosophic semi-generalized continuous in PNTS. We also contribute nets, filters, and borders to this pentapartitioned neutrosophic concept. 
ISSN: 2456-8686, 5(1), 2021:123-131

https://doi.org/10.26524/cm99

\section{Funding: This research received no external funding}

Conflicts of Interest: The authors declare no conflict of interest.

\section{References}

[1] Ahmed B, AL-Nafee, Said Broumi,F.Smarandache, Neutrosophic soft bitopological spaces, International Journal of Neutrosophic Science, 14(1), 47-56 (2021).

[2] Arockiarani I, Dhavaseelan R, Jafari S, Parimala M, On some notations and functions in neutrosophic topological spaces, Neutrosophic sets and systems, 16, 16-19 (2017).

[3] Arockiarani I , Sumathi I R and Martina Jency J, Fuzzy neutrosophic soft topological spaces, IJMA, 4(10), 225-238 (2013).

[4] Mullai M , Said Broumi, Dominating Energy in Neutrosophic Graphs, International Journal of Neutrosophic Science, 5, 38-58 (2020).

[5] Radha R, Stanis Arul Mary A, Smarandache F, Pentapartitioned Neutrosophic pythagorean Soft set, IRJMETS, 3(2), 905-914 (2021).

[6] Radha, Stanis Arul Mary A, Pentapartitioned Neutrosophic Pythagorean Ser, IRJASH, 3, 62-82 (2021).

[7] Radha R, Stanis Arul Mary A, Heptapartitioned neutrosophic sets, IRJCT, $2,222-230,(2021)$

[8] Radha R, Stanis Arul Mary A, F.Smarandache, Quadripartitioned Neutrosophic Pythagorean soft set, International Journal of Neutrosophic Science, 14(1), 9-23 (2021).

[9] Radha R, Stanis Arul Mary A, F.Smarandache, Neutrosophic Pythagorean soft set, Neutrosophic sets and systems, 42, 65-78 (2021).

[10] Radha R, Stanis Arul Mary A, Pentapartitioned Neutrosophic Semi-open sets" (Submitted).

[11] Rama Malik, Surapati Pramanik, Pentapartitioned Neutrosophic set and its properties, Neutrosophic Sets and Systems, 36, 184-192 (2020). 
ISSN: 2456-8686, 5(1), 2021:123-131

https://doi.org/10.26524/cm99

[12] Salama A and Al - Blowing S A, Neutrosophic Set and Neutrosophic topological spaces, IOSR Journal of Math., 4, 31 - 35 (2012).

[13] Smarandache F, Degree of Dependence and independence of the sub components of fuzzy set and neutrosophic set, Neutrosophic sets and systems, 11, 95-97 (2012).

[14] Smarandache F, Neutrosophy and Neutrosophic Logic, First International Conference on Neutrosophy, Neutrosophic Logic, Set, Probability and Statistics University of New Mexico, Gallup, NM 87301, USA, 5-21 (2002).

[15] Smarandache F,Said Broumi, True -False set is a particular case of the refined Neutrosophic set, International Journal of Neutrosophic Science, 12(1), 9-12 (2020).

[16] Smarandache F, Neutrosophic set, A generialization of the intuituionistics fuzzy sets, Inter. J. Pure Appl. Math., 24 , 287-297 (2005).

[17] Xindong Peng, Yong Yang, Some results for pythagorean fuzzy sets, International Journal of Intelligent systems, 30, 1133-1160 (2015).

[18] Zadeh L A, Fuzzy Sets, Inform and Control, 8, 338 -353 (1965).

[19] Zhaowen Li, Rongchen cui, On the topological structure of intuitionistic fuzzy soft sets, Annals of Fuzzy Mathematics and Informatics, 5(1), 229-239 (2013). 